\title{
Two-dimensional simulation of an electron cyclotron resonance plasma source with self-consistent power deposition
}

\author{
Minghai Liu ${ }^{\mathrm{a}, \mathrm{b}, *}$, Xiwei $\mathrm{Hu}^{\mathrm{c}}$, Hanming $\mathrm{Wu}^{\mathrm{d}}$, Qinchong $\mathrm{Wu}^{\mathrm{b}}$, Guoyang $\mathrm{Yu}^{\mathrm{b}}$, \\ Yuan $\operatorname{Pan}^{\mathrm{a}}$ \\ ${ }^{a}$ Department of Electrical Engineering, Huazhong University of Science and Technology, Wuhan, Hubei 430074, PR China \\ ${ }^{\mathrm{b}}$ Institute of Plasma Physics, Chinese Academy of Sciences, Hefei, Anhui 230031, PR China \\ ${ }^{\mathrm{c}}$ Department of Modern Physics, University of Science and Technology of China, Hefei, Anhui 230031, PR China \\ ${ }^{\mathrm{d}}$ Institute of Mechanics, Chinese Academy of Sciences, Beijing 100080, PR China
}

\begin{abstract}
Using a refined two-dimensional hybrid-model with self-consistent microwave absorption, we have investigated the change of plasma parameters such as plasma density and ionization rate with the operating conditions. The dependence of the ion current density and ion energy and angle distribution function at the substrate surface vs. the radial position, pressure and microwave power were discussed. Results of our simulation can be compared qualitatively with many experimental measurements. (C) 2000 Published by Elsevier Science B.V. All rights reserved.
\end{abstract}

PACS: 52.65.-y; 52.50. Fi; 52.80. Pi; 52.65. Pp; 52.65. Rr

Keywords: Electron cyclotron resonance; Microwave; Plasma; Density; Ionization

\section{Introduction}

Progress in the fabrication of microelectronics requires more stringent technical developments in plasma processing [1]. Over the past several years, electron cyclotron resonance (ECR) plasma has become one of the promising techniques which meets these needs [2]. The higher plasma density produced by ECR plasma sources at low pressure makes them particularly attractive for application in etching as well as in thin film deposition. The current developing tendency is the requirement of a uniform plasma over a large area with less damage of the wafer by ion impacting energy [3]. Therefore, many experimental works [16-18] have been

\footnotetext{
* Corresponding author. Department of Electrical Engineering, Huazhong University of Science and Technology, Wuhan, Hubei 430074, PR China.
}

done to understand the performance of ECR plasma sources and the properties of corresponding plasma processing. However, in spite of the extensive research on processes in ECR plasma, many phenomena that have been observed in ECR plasma sources are still not well understood. For many processing applications, the optimization of operation is carried out on an empirical scale. However, there is an increasing awareness that plasma models and simulations could be used as powerful tools in increasing the general understanding of plasma processing, giving insights into some discharge parameters which are not easily accessible to laboratory measurements. Recent progress along these lines has been impressive and much more effort has been made to develop computer simulation models of ECR plasma sources. The kinetic model [4], the fluid models $[5,6]$ and the hybrid model [7-10] in one or two dimensions have been presented sequentially. In many 
experimental and numerical works, the ion current density and ion energy distribution function(IEDF) at the substrate surface which are directly related to the quality of plasma processing are of especially concern. This article discusses not only the change of plasma parameters such as plasma density and ionization rate with operation conditions, but also ion current density and ion energy and angle distribution function at the substrate surface in an extended electron cyclotron resonance plasma source.

\section{The design features and physical model}

\subsection{The design features of the ECR reactor}

The ECR reactor used for simulation was identical to that described by Zhan [11]. A microwave $(2.45 \mathrm{GHz})$ was supplied to the vacuum chamber $(16 \mathrm{~cm}$ in diameter, $33 \mathrm{~cm}$ in length) through a quartz window $(10 \mathrm{~cm}$ in diameter, $18 \mathrm{~cm}$ in length). The divergent magnetic field was established by two external coils. The axial position of resonant zone was approximately $9-13 \mathrm{~cm}$. The wafer was located on the bottom of the chamber. The reactor walls were grounded except the microwave dielectric window. Argon was used as the working gas.

\subsection{Physical model}

Our physical model is a fluid electron-particle ion hybrid model in two-dimensional cylindrical axisymmetric geometry. Its characteristics have been described in detail elsewhere [7-10], so the following description is quite brief.

\subsubsection{Electron description}

Electron is modeled as a fluid with mass conservation

$\nabla \cdot \overrightarrow{j_{\mathrm{e}}}=R_{\text {ion }}\left(n_{\mathrm{e}}, n_{\mathrm{i}}, n_{0}\right)$

$\overrightarrow{j_{\mathrm{e}}}=\mu\left[n_{\mathrm{e}} \nabla \phi-\nabla\left(n_{\mathrm{e}} T_{\mathrm{e}}\right) / \mathrm{e}\right]$

energy conservation

$\vec{Q}=2 k T_{\mathrm{e}}\left(\overrightarrow{j_{\mathrm{e}}}-\mu n_{\mathrm{e}} \nabla T_{\mathrm{e}} / \mathrm{e}\right)$

$\nabla \vec{Q}=P_{\text {ecr }}+\mathrm{e} \overrightarrow{j_{\mathrm{e}}} \cdot \nabla \phi-P_{\text {loss }}$

and Poisson equation

$\varepsilon_{0} \Delta \phi=\mathrm{e}\left(n_{\mathrm{e}}-n_{\mathrm{i}}\right)$

where $n_{\mathrm{e}}, n_{\mathrm{i}}, n_{0}, \phi, T_{\mathrm{e}}, \overrightarrow{j_{\mathrm{e}}}, \vec{Q}, \mu, R_{\text {ion }}$ and $\varepsilon_{0}$ are electron density, ion density, neutral density, electrostatic potential, electron temperature, electron flux, electron energy flux, mobility, ionization rate and permeability, respectively. $P_{\text {loss }}$ and $P_{\text {ecr }}$ are the power consumption for various loss mechanisms and the microwave power absorption, respectively.

\subsubsection{Ion description}

Ions are considered as individual particles and their dynamic behavior is described by combining a particlein-cell and Monte Carlo code in two spatial dimensions $(r, z)$ and three velocity space dimensions $\left(v_{r}, v_{\theta}, v_{z}\right)$ when they move into the applied magnetic field and self-consistent electrostatic field. Ions are generated by the ionization or charge exchange event.

\subsubsection{Boundary conditions}

The sheath in the model is assumed infinitesimally thin. The potential discontinuity between plasma sheath and reactor wall is defined as $\Delta \phi=\phi_{\mathrm{s}}-\phi_{\mathrm{w}}$. In a Maxwellian electron velocity distribution, the magnitude of the electron flux to the wall is

$j_{\text {ew }}=n_{\text {es }} v_{\mathrm{es}} \exp \left[\left(-\mathrm{e} \Delta \phi / k T_{\mathrm{es}}\right)\right] / 4$

where $T_{\mathrm{e}}, n_{\mathrm{es}}$ and $\nu_{\mathrm{es}}$ are electron temperature, electron density, and average thermo-velocity at the plasma-sheath boundary, respectively. The electron energy flux is represented analogously by

$Q_{\text {ew }}=k T_{\text {es }} j_{\mathrm{ew}}\left[2+\mathrm{e} \Delta \phi / k T_{\mathrm{es}}\right]$

The second kind of the boundary is of dielectric wall, where the surface potential will float with respect to ground. The wall potential $\phi_{\mathrm{w}}$ is determined by the balance of the ion and electron current density $j_{\text {iw }}\left(=j_{\text {ew }}\right)$ at each grid point of boundary:

$\phi_{\mathrm{w}}=\phi_{\mathrm{s}}+k T_{\mathrm{e}} / \mathrm{e} \ln \left[4 j_{i w} /\left(n_{\mathrm{es}} v_{\mathrm{th}}\right)\right]$

Recently, some corrections such as self-consistent treatment of neutral particle density, the influence of electron-ion collision are included in this model by $\mathrm{Wu}$ [8] and Cronrath [12] respectively. In all these publications [7-10,12], the microwave power absorption is always assumed as a fixed functional form which neglected plasma effect. However, many experiments indicated that the microwave power absorption is strongly dependent on plasma parameters at the resonant zone. This showed that the simulation of ECR plasma has to be performed in a self-consistent manner. Therefore, we made an important improvement on the previous physical model. In the refined model, the microwave power absorption $P_{\text {ecr }}$ at resonant regions has been treated self-consistently with plasma kinetic theory. For the sake of brevity, the details of treatments are not 
given, but can be found in Liu et al. [13,14]. Here we present only the finally expression of microwave power absorption at the resonance zone with the assumption of a Maxwellian electron distribution function and a negligible vertical component of wave vector:

$$
\begin{aligned}
P_{\mathrm{ecr}}= & \operatorname{Im}\left\{2 \frac{\omega_{\mathrm{p}}^{2}}{k_{\|}^{2} v_{\mathrm{T}}^{2}} \varepsilon_{0}\left[1+\frac{\omega}{k_{\|} \nu_{\mathrm{T}}} Z_{\mathrm{p}}\left(\frac{\omega}{k_{\|} \nu_{\mathrm{T}}}\right)\right]\right\}\left|E_{z}\right|^{2} \\
& +\operatorname{Im}\left\{\sqrt{2} \varepsilon_{0} \frac{\omega_{\mathrm{p}}^{2}}{k_{\|}^{-} \nu_{\mathrm{T}}} Z_{\mathrm{p}}\left(\frac{\omega+\omega_{\mathrm{c}}}{k_{\|}^{-} \nu_{\mathrm{T}}}\right)\right\}\left|E_{-}\right|^{2} \\
& +\operatorname{Im}\left\{\sqrt{2} \varepsilon_{0} \frac{\omega_{\mathrm{p}}^{2}}{k_{\|}^{+} \nu_{\mathrm{T}}} Z_{\mathrm{p}}\left(\frac{\omega-\omega_{\mathrm{c}}}{k_{\|}^{+} \nu_{\mathrm{T}}}\right)\right\}\left|E_{+}\right|^{2}
\end{aligned}
$$

These terms at the right-hand side represent the contribution of the Landau damping, the anomalous Doppler damping and the cyclotron damping sequentially, where $\varepsilon_{0}, \nu_{\mathrm{T}}, \omega, \omega_{\mathrm{p}}, \omega_{\mathrm{c}}$ are permeability, thermal velocity of electron, microwave frequency, plasma frequency and electron cyclotron resonance frequency, respectively. $E_{\mathrm{z}}$ is the axial component of the microwave electric field, $\mathrm{E}_{-}$and $E_{+}$are the component of electric field of the left- and right-hand polarized wave, respectively, $k_{\|}, k_{\|}^{-}$, and $k_{\|}^{+}$are the corresponding wave vectors. These quantities are related with the propagation of the microwave waves in the resonant cavity chamber, and discussed in Liu et al. [15]. It is obvious that the present microwave power deposition profiles depend automatically on the changes of plasma parameters.

\section{Simulation results}

\subsection{Plasma density}

Fig. 1 illustrates the dependence of plasma density of TM mode both in the source area and the downstream region on the neutral gas pressures. It can be seen that plasma density grows with pressure at low pressure (below 6 mtorr), at higher pressure, plasma density slowly decreases. This result is observed in many experimental measurements [16]. This can perhaps be attributed to the complicated dependence of ionization rates on pressure and the imbalance of ionization and loss of particle.

\subsection{Ion energy and angle distribution function at substrate surface}

\subsubsection{Ion energy distribution function vs. pressure at the substrate surface}

The dependence of ion energy distribution function (IEDF) of TE mode vs. pressure at the substrate sur-

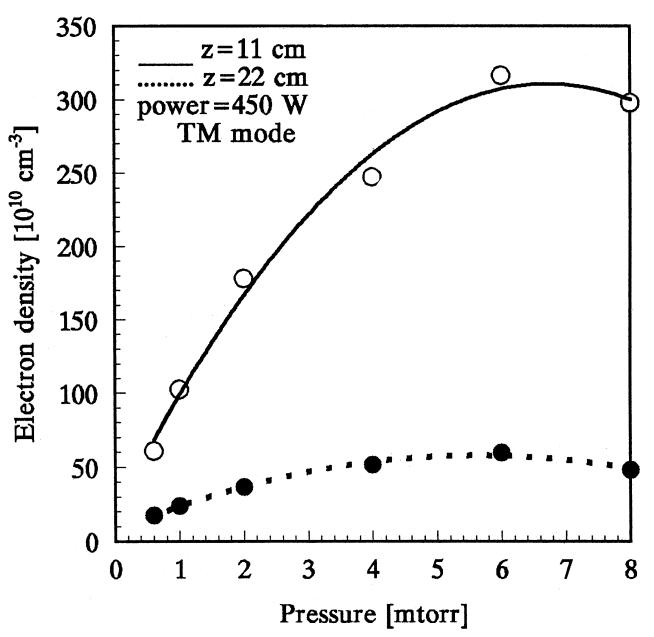

Fig. 1. The dependence of plasma density of TM mode vs. pressure.

face is shown in Fig. 2. The average energy, the probable energy and the tail portion of these distributions increase with decreasing pressure. These results can be contributed to the decrease of the ion-neutral collision and the increase of plasma potential in the low pressure.

\subsubsection{Ion angle distribution function vs. position at the substrate surface}

When an ion moves towards the substrate, its impact angle is generally not perpendicular to the surface. Defining the ion impact radial pitch angle as $\operatorname{arctg}\left(v_{r} / \nu_{z}\right)$, the ion impact radial angle distributions of TE mode for various radial positions are plotted in Fig. 3. These distributions peak at larger angles at larger radial position, reflecting the radial ion drift motion following the diverging magnetic field lines. It is interested that all the full width at half-maximum (FWHM) of the distributions increase with the radial positions.

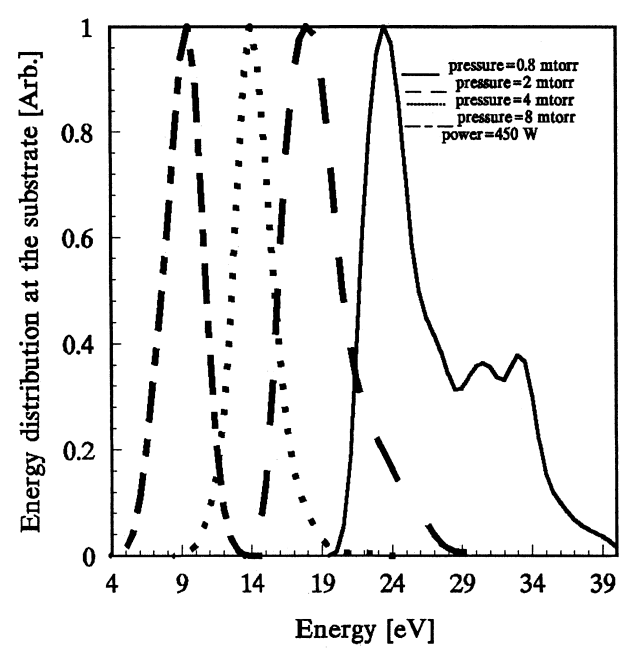

Fig. 2. The dependence of ion energy distribution function of TE mode vs. pressure at substrate surface $(32<z<33 \mathrm{~cm}, 0<r<2 \mathrm{~cm})$. 


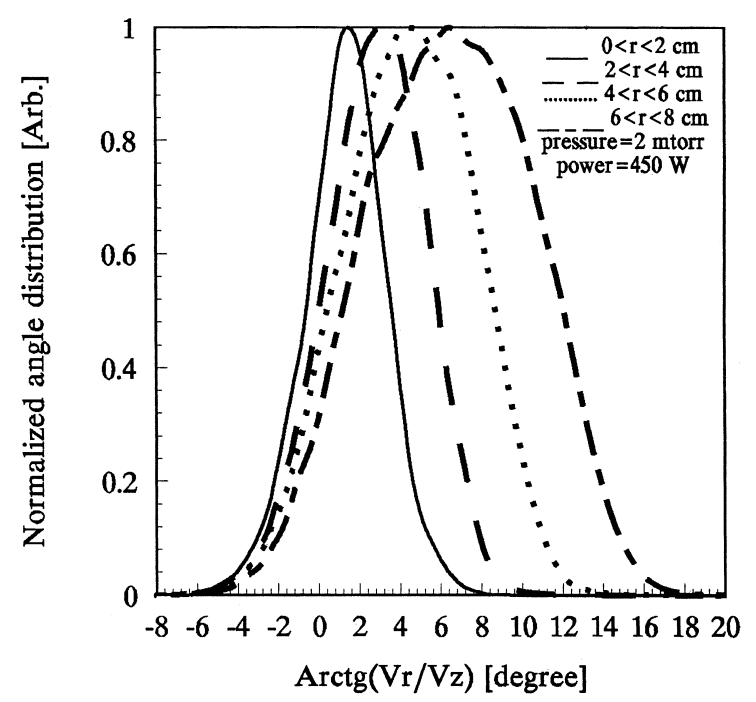

Fig. 3. The dependence of ion radial angle distribution function of TE mode vs. radial position at substrate surface $(32<z<33 \mathrm{~cm})$.

\subsubsection{Ion angle distribution function at the substrate surface}

The dependence of ion radial angle distribution function of TM mode and ion azimuthal angle distribution function of TE mode vs. pressure at the substrate surface is shown in Figs. 4 and 5, respectively. It is obvious that a lower pressure results in a smaller angle and a narrowed FWHM of the distribution. The results can be interpreted as follows: there is higher plasma potential in lower pressure, so ions obtain more energy from the electrostatic field. However, less collision of neutral ions in low pressure results in a less energy transfer from the parallel direction to the perpendicular direction.

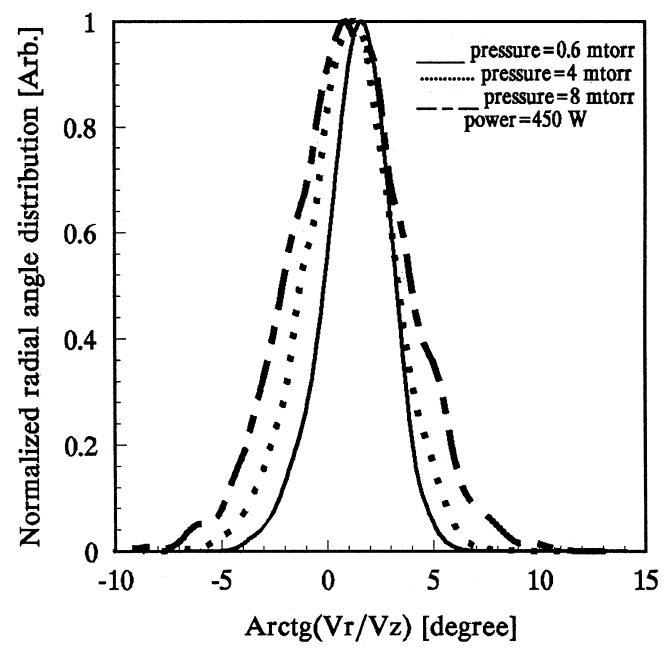

Fig. 4. The dependence of ion radial angle distribution function of TM mode vs. pressure at substrate surface $(32<z<33 \mathrm{~cm}, 0<r<1$ $\mathrm{cm})$.

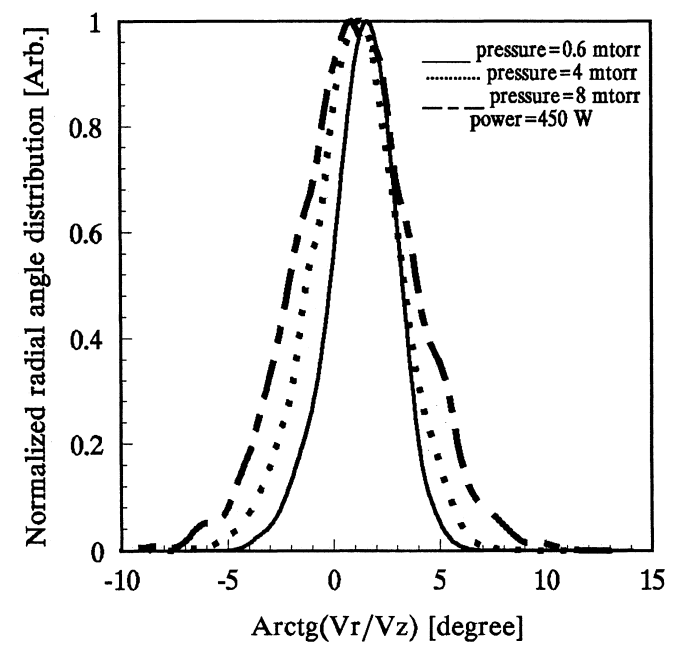

Fig. 5. The dependence of ion azimuthal angle distribution function of TE mode vs. power at substrate surface. The positions are same as shown in Fig. 4.

\subsection{Ion current density at the substrate surface}

The ion current density and its uniformity are key in determining the performance of plasma processing. Ion current density towards the substrate surface can be estimated through the approximate expression [17] $J \approx$ $\mathrm{e} n_{\mathrm{w}} c_{\mathrm{s}}$, where $n_{\mathrm{w}}$ is ion density close the wafer, $c_{\mathrm{s}}=$ $\left(k T_{\mathrm{e}_{\mathrm{w}}} / M_{\mathrm{i}}\right)^{0.5}$ is the Bohm velocity. The variation of ion current density of the TE mode with pressure at the substrate surface is given in Fig. 6. It can be seen that the ion current densities increase with pressure in low pressure and decrease greatly at higher pressure. On the other hand, its non-uniformity increases gradually with pressure. These features indicate that the choice of pressure is very important for obtaining high processing rate with good uniformity. The dependence of

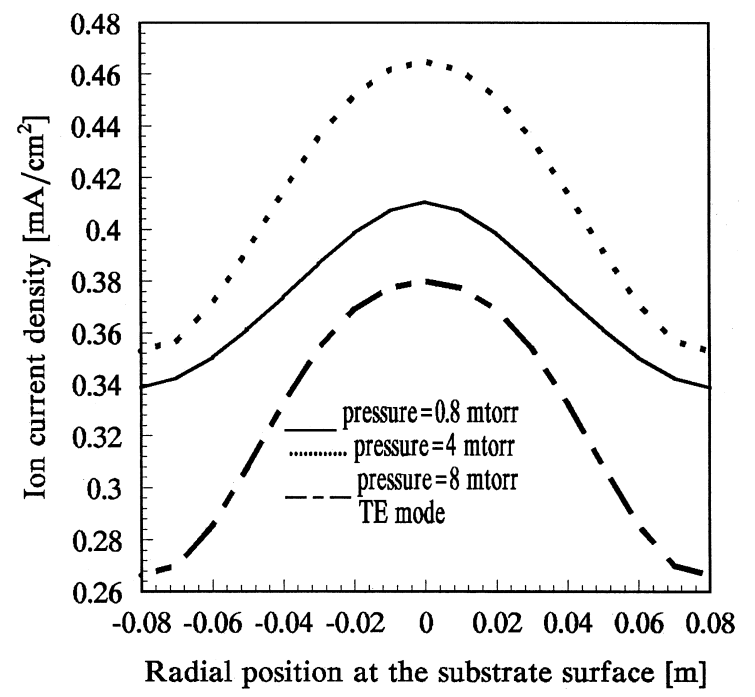

Fig. 6. The ion current density of TE mode vs. pressure at the substrate surface $(32<z<33 \mathrm{~cm}$, power $=450 \mathrm{~W})$. 


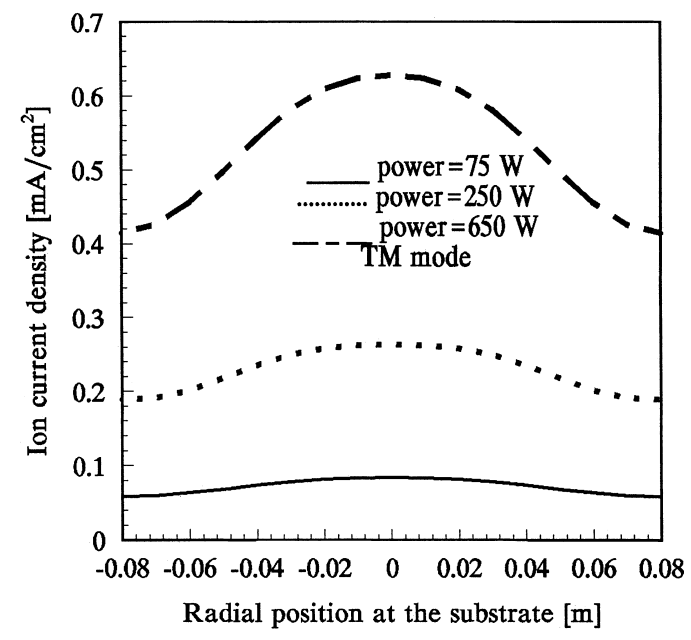

Fig. 7. The ion current density of TM mode vs. power at the substrate surface $(32<z<33 \mathrm{~cm}$, pressure $=8 \mathrm{mtorr})$.

ion current density of TM mode vs. microwave power at the substrate surface is plotted in Fig. 7. The ion current density increase with microwave power, at the same time, its uniformity becomes poor also. These simulation results are qualitatively in good agreement with many experimental observations [17-19].

\section{Conclusions}

In this paper, we presented a refined two-dimensional hybrid-model with self-consistent microwave absorption to investigate the change of plasma parameters such as plasma density and ionization rate with the operating conditions. The dependencies of the ion current density and ion energy and angle distribution function at the substrate surface vs. the radial position, pressure and microwave power were discussed. The main conclusions can be drawn as follows:

1. Plasma density increases with pressure at low pressure, and decreases at higher pressure.

2. The average energy, the probable energy and the tail portion of ion energy distribution function increase with decreasing pressure.

3. The average angle, the probable energy and the FWHM of the IADF distribution increase with the radial position.

4. A lower pressure results in a smaller angle and a narrowed FWHM of the IADF distribution.
5. The ion current density increases with pressure in low pressure and decreases at high pressure, and increases with microwave power. Its uniformity becomes poorer at higher pressure and power.

\section{Acknowledgements}

This work was supported by the National Nature Science Foundation of China and by the Postdoctoral Science Foundation of China. Appreciation is also expressed to Professor Y.Y. Li for his stimulating discussions and Professor R.J. Zhan and Professor J.X. Ma for their encouragement and useful advice.

\section{References}

[1] J. Asmussen, J. Vac. Sci. Technol. A 7 (1989) 883.

[2] Z.Y. Ning, Z.X. Ren, Progress in Physics 12 (1992) 38 ((in Chinese)).

[3] F.F. Chen, Physics Plasma 2 (1995) 2164.

[4] M.A. Hussein, G.A. Emmert, J. Vac. Sci. Technol. A 8 (1990) 2913.

[5] G. Guan, M.E. Mauel, W.M. Holber, J.B.O. Caughman, Phys. Fluids B 4 (1992) 4177.

[6] H. Nihei, Jpn J. Appl. Phys. 32 (1993) 2105.

[7] P.K. Porteous, H.M. Wu, D.B. Graves, Plasma Source Sci. Technol. 3 (1994) 25.

[8] H.M. Wu, D.B. Graves, M. Kilgore, Plasma Source Sci. Technol. 6 (1997) 231.

[9] M.H. Liu, X.W. Hu, H.M. Wu, J. Appl. Phys. 81 (1997) 7734.

[10] M.H. Liu, X.W. Hu, H.M. Wu, Nucl. Fusion Plasma Phys. 18 (1998) 36 ((in Chinese)).

[11] R.J. Zhan, C.Y. Wang, X.H. Wen, X.D. Zhu, Plasma Coating Technol. 105 (1998) 72.

[12] W. Cronrath, N. Mayumi, M.D. Bowden, K. Uchino, K. Muraoka, J. Appl. Phys. 82 (1997) 1036.

[13] M.H. Liu, Doctoral dissertation, Department of Modern Physics: University of Science and Technolnology, Hefei, China, 1997 ((in Chinese)).

[14] M.H. Liu, X.W. Hu, Q.C. Wu, G.Y. Yang, Chin. Phys. Lett. 17 (1) $(2000) 31$.

[15] M.H. Liu, X.W. Hu, H.M. Wu, Q.C. Wu, G.Y. Yu, Z.X. Ren, J. Appl. Phys. 87 (3) (2000) 1070.

[16] M.H. Francombe, J.L. Vossen, Physics of Thin Film, Academic Press, New York, 1994.

[17] D.A. Carl, M.C. Willamson, M.A. Lieberman, A.J. Lichtenberg, J. Vac. Sci. Technol. B 9 (1991) 339.

[18] R.A. Dandl, G.E. Guest, J. Vac. Sci. Technol. A 9 (1991) 3119.

[19] S.M. Gorbakin, L.A. Berry, J.B. Roberto, J. Vac. Sci. Technol. A 8 (1990) 2893. 